\title{
Digital Chaotic Synchronized Communication System
}

\author{
S.G. Stavrinides ${ }^{* 1}$, A.N. Anagnostopoulos ${ }^{1}$, A.N. Miliou $^{2}$, A. Valaristos ${ }^{2}$, \\ L. Magafas ${ }^{3}$, K. Kosmatopoulos ${ }^{1}$ and S. Papaioannou ${ }^{4}$
}

\author{
(1) Physics Department, Aristotle University of Thessaloniki, 54124, Thessaloniki, Greece. \\ (2) Department of Informatics, Aristotle University of Thessaloniki, 54124, Thessaloniki, Greece. \\ (3) Department of Electrical Engineering, Technological and Educational Institute of Kavala, Kavala, Greece. \\ (4) Department of Civil Engineering, Technological and Educational Institute of Serres, Serres, Greece.
}

Received 19 March 2009; Revised 16 June 2009; Accepted 12 July 2009

\begin{abstract}
The experimental study of a secure chaotic synchronized communication system is presented. The synchronization between two digital chaotic oscillators, serving as a transmitter-receiver scheme, is studied. The oscillators exhibit rich chaotic behavior and are unidirectionally coupled, forming a master-slave topology. Both the input information signal and the transmitted chaotic signal are digital ones.
\end{abstract}

Keywords: Nonlinear circuits, Chaotic circuits, Digital chaotic oscillator, Synchronization.

\section{Introduction}

Nonlinear oscillator synchronization is a process that is frequently encountered in nature, explaining relevant phenomena. A significant property that nonlinear dynamical systems possess is their ability to be synchronized. As a consequence, chaotic system synchronization is encountered in a variety of scientific fields, from astronomy and electronic engineering to social sciences.

Chaotic deterministic signals exhibit several intrinsic features, beneficial to secure communication systems, both analog and digital ones. Two key features of deterministic chaos are the "noise-like" time series and the sensitive dependence on initial conditions $[1,2]$. Both of them grant to chaotic signals low probability of detection in chaotic transmissions and low probability of decoding, in case of interception [3].

Due to their possible application for secure internet communications, a number of promising non-linear circuits, demonstrating chaotic behavior, have been presented in the last decade [4-8]. There are two main issues in studying the control of chaotic electronic circuits suitable for secure communications [9]. The first one is the way a non-linear circuit begins to operate in chaotic mode (route to chaos) $[2,10,11]$ and the second one is the achievement of synchronization between transmitter-receiver [12, 13].

Since the discovery by Pecora and Carroll that chaotic systems can be synchronized [12], the topic of synchronization of coupled chaotic circuits and systems has been studied intensely [14] and some interesting applications such as broadband communication systems or cryptographic systems have come out of

* E-mail address: stavros@physics.auth.gr ISSN: 1791-2377 (C) 2009 Kavala Institute of Technology. All rights reserved. this research [15-18].

In this paper the system synchronization properties of a chaotic communication system, suitable for secure communication, are examined.

\section{Scheme and Circuit description}

A very interesting electronic circuit exhibiting chaotic behavior and with potential applications in secure communications, was proposed and numerically examined in [19], while its transmitter has been experimentally studied in [20]. The circuit, of the system under question, is presented in Fig 1.

Both the transmitter and receiver, of this chaotic communication system, are second order non-linear non-autonomous electronic circuits, with their mode of operation depending on the externally applied driving frequency. It has already been found that the transmitter circuit exhibits the period doubling [21, 22] and the intermittency [23, 24] routes to chaos as well as internal crisis [25], in different ranges of driving signal frequency $M(t)$.

The main advantage of this circuit is that it is capable of synchronized chaotic communication, suitable for transmission of digital signal. It should be noted that the transmitted chaotic signal is not analog but a discrete one. Moreover, there is no need of transmitting any special synchronization signal. Synchronization is achieved by the transmitted chaotic information (discrete) signal, itself.

The transmitter and the receiver are identical circuits $[19,20]$. 
Both the circuits include an integrator-based second-order RC resonance loop, a comparator $\mathrm{H}$ (the circuit's non-linear element), an exclusive OR gate, with an input $M(t)$, for the external source and a buffer to avoid overloading of the XOR gate. The external excitation $M(t)$, that is necessary for non-autonomous oscillators, can be either a sequence of square pulses of period $\mathrm{T}=2 \pi / \omega$ or a more complex signal, if one wants to encode an arbitrary message, for example. This external excitation serves as the system's information signal.

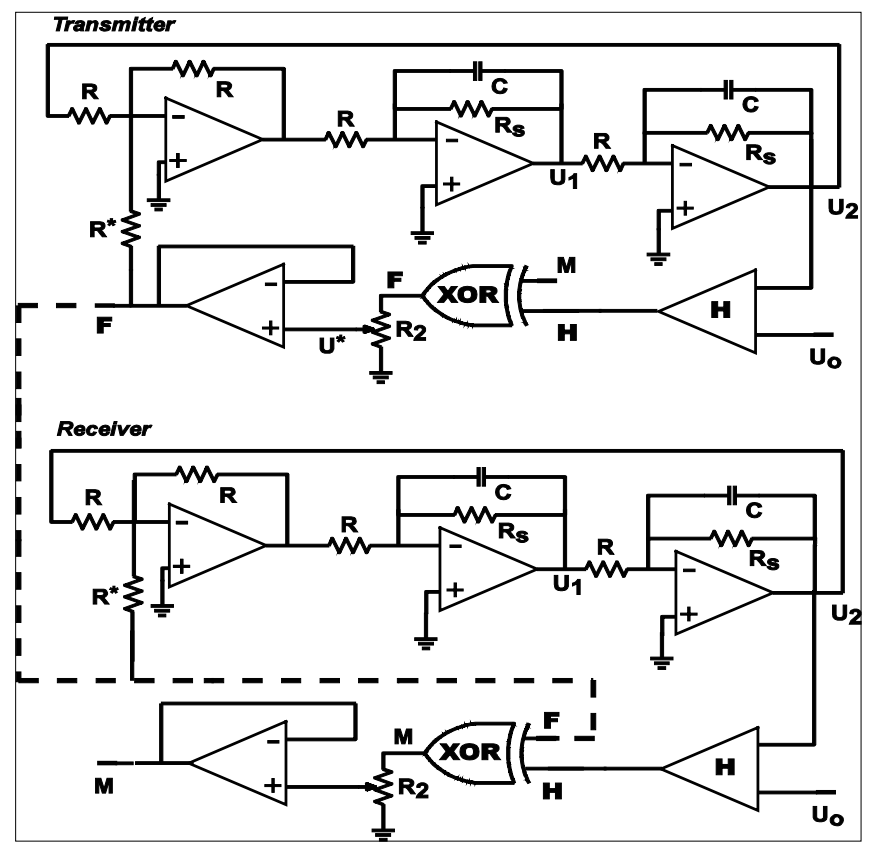

Figure 1. Schematic diagram of the transmitter-receiver system

The main target is the exact reconstruction, at receiver's output, of information signal $M(t)$ applied at the transmitter circuit. This is achieved by synchronously reconstructing $U_{2}$ at the transmitter's-receiver's analog output.

The principle of operation is demonstrated below. Here the chaotic pulses $U^{*}(t) \propto F\left(y_{1}, t\right)$ drive the resonance loops (analog part) of both the transmitter and the receiver. The transmitter system is governed by the following set of equations:

$$
\begin{aligned}
& \dot{x}_{1}=a F\left(y_{1}, t\right)-b x_{1}+y_{1} \\
& \dot{y}_{1}=-x_{1}-b y_{1}
\end{aligned}
$$

$$
F\left(y_{1}, t\right)=H\left(y_{1}\right) \oplus M(t)
$$

while the receiver is governed by:

$$
\dot{x}_{2}=a F\left(y_{1}, t\right)-b x_{2}+y_{2}
$$

$\dot{y}_{2}=-x_{2}-b y_{2}$

It should be noted the same driving term $\alpha F\left(y_{1}, t\right)$, in equations (1a) for the transmitter and (2a) for the receiver, which represents the system's coupling factor. The following substitutions have been used in the previous systems of equations, since the parameters are written in a dimensionless form:

$$
\begin{aligned}
& \mathrm{x}=\frac{U_{1}}{U_{o}}, \quad y=\frac{U_{2}}{U_{o}}, \quad t=\frac{1}{R C} \\
& \alpha=\frac{U^{*} \cdot R}{U_{o} \cdot R^{*}}, \quad b=\frac{R}{R_{s}} \\
& \omega=\omega_{M} R C
\end{aligned}
$$

The symbol $\oplus$ stands for the XOR operation, while $H^{S}$ stands for the shifted Heaviside function $H^{S}(y)=H(-y+1) . M(t)$ is the normalized square pulse input signal of period $T=2 \pi / \omega_{1}$.

The circuit demonstrates only damped oscillations, as long as no excitation is applied to the XOR gate. The amplitude of the oscillating variables $U_{1}$ and $U_{2}$ converges exponentially ( $\propto e^{-b t}$ ) to a stable steady state, for all reasonable initial conditions $\left(U_{1}^{2}+U_{2}^{2}<U_{o}^{2}\right.$ or $\left.x^{2}+y^{2}<1\right)$ while for a non zero external drive $M(t)$ the circuit becomes periodically forced, exhibiting chaos.

Introducing in the set of equations (1), the error variables $\Delta x=x_{2}-x_{1}$ and $\Delta y=y_{2}-y_{1}$, we obtain the equations governing the error dynamics:

$$
\begin{aligned}
& \Delta \dot{x}=b \Delta x+\Delta y \\
& \Delta \dot{y}=-\Delta x-b \Delta y
\end{aligned}
$$

The solution of (4) shows the exponential decrease of the errors for all possible initial errors $\Delta \mathrm{x}_{\mathrm{o}}$ and $\Delta \mathrm{y}_{\mathrm{o}}$. Thus, the synchronization is globally asymptotically stable. This requirement leads to the conclusion that for $\Delta \mathrm{x} \rightarrow 0$ and $\Delta \mathrm{y} \rightarrow 0$, the corresponding state variables, are robustly synchronized $\left(\mathrm{x}_{1} \rightarrow \mathrm{x}_{2}\right.$ and $\left.\mathrm{y}_{1} \rightarrow \mathrm{y}_{2}\right)$. Consequently, the non-linear functions behave in a synchronous way $\mathrm{H}\left(\mathrm{y}_{2}\right) \rightarrow \mathrm{H}\left(\mathrm{y}_{1}\right)$ as well.

This result suggests an extremely simple technique of recovering the signal $M(t)$ at the receiver end. The received signal is applied to the XOR unit of the receiver. Due to the sum $\bmod 2$ property, the signal $F\left(y_{1}, t\right)$ can be recovered from the chaotic one without any errors, according to:

$$
F\left(y_{1}, t\right) \oplus H\left(y_{2}\right)=H\left(y_{1}\right) \oplus M(t) \oplus H\left(y_{2}\right) \rightarrow \rightarrow H\left(y_{1}\right) \oplus M(t) \oplus H\left(y_{1}\right)=M(t)(5)
$$

\section{Experimental Results}

Synchronization between transmitter and receiver was experimentally verified. Both sub-circuits remained synchronized under different conditions, regarding the circuit parameters, as well as, the driving frequency $f_{M}$, which was provided by a digital signal generator (HM8130). It should be noted that the driving frequency represents the external digital information that is fed to the communication system.

In this section a typical modulation-demodulation procedure through chaotic synchronization is presented. All signals were monitored by a digital storage oscilloscope (HP54603B), further 
connected to a PC for recording and analysis purposes, so that the proper characterization of the circuit behavior could be achieved. Appropriate software, built in NI's LabView environment, was used in order to control all digital instruments used and process the signals acquired [26].

The system's parameters were set to be equal in both the transmitter and the receiver circuits. In order to operate in a chaotic mode, the parameter values, for $U_{\mathrm{o}}=350 \mathrm{mV}$ and $U^{*}=4 \mathrm{~V}_{\mathrm{p}-\mathrm{p}}$, were set at $\alpha=6.35$ and $b=0.02$. For this set of parameter values, the system exhibits chaotic behavior in various ranges of external excitation $f_{M}$ (chaotic windows) and undergoes various routes to chaos [21-25].

In order to study the system's synchronization while it operates in a chaotic mode, the driving frequency was set to $f_{M}=6,222$ $K H z$. In Fig. 2 the transmitter's phase portrait $\left(U_{1}\right.$ vs. $\left.U_{2}\right)$ is presented. The chaotic nature of signals $U_{1}$ and $U_{2}$ is evident. Next to the phase portrait, the transmitter's chaotic characterization has been already confirmed in [20].

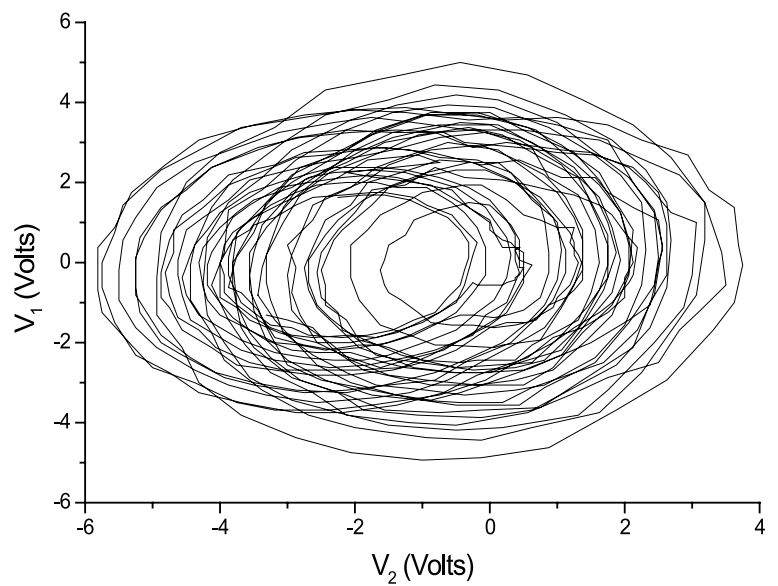

Figure 2. The phase portrait $U_{1}-U_{2}$ characterizing the transmitter's chaotic operation.

In Fig. 3 the driving pulse signal $M(t)$ is shown, together with its power spectrum. The periodic nature of $M(t)$ is obvious. In Fig. 4(a) the signal $F\left(y_{1}, t\right)$ at the transmitter's digital output appears, while in Fig 4(b) the corresponding power spectrum is shown. The power spectrum confirms the wideband nature of the output signal. This wideband signal shows the impossibility of detecting the original information by using simple filtering processes.

(a)

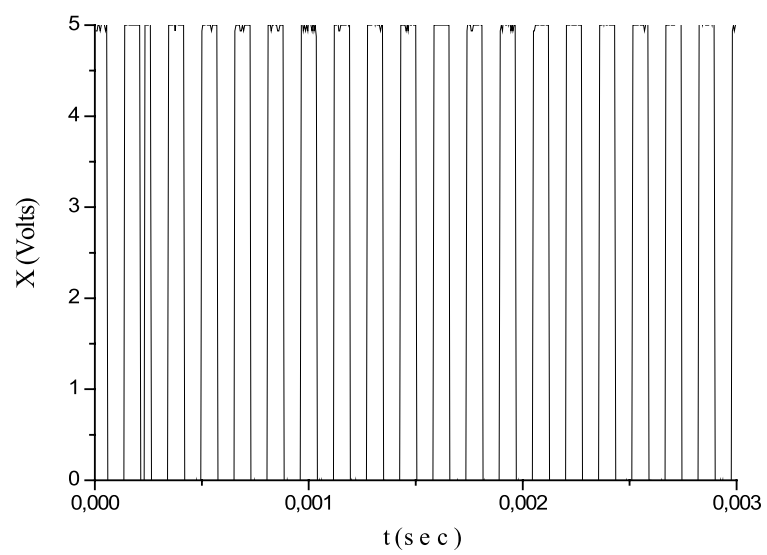

According to $[27,28]$, one can conclude that the present chaotic communication scheme can be secure. Finally, in Fig. 5(a) the recovered signal is presented. Both the signals itself, as well as, the corresponding power spectrum - Fig. 5(b) - demonstrate the fact that the recovery procedure is quite exact.

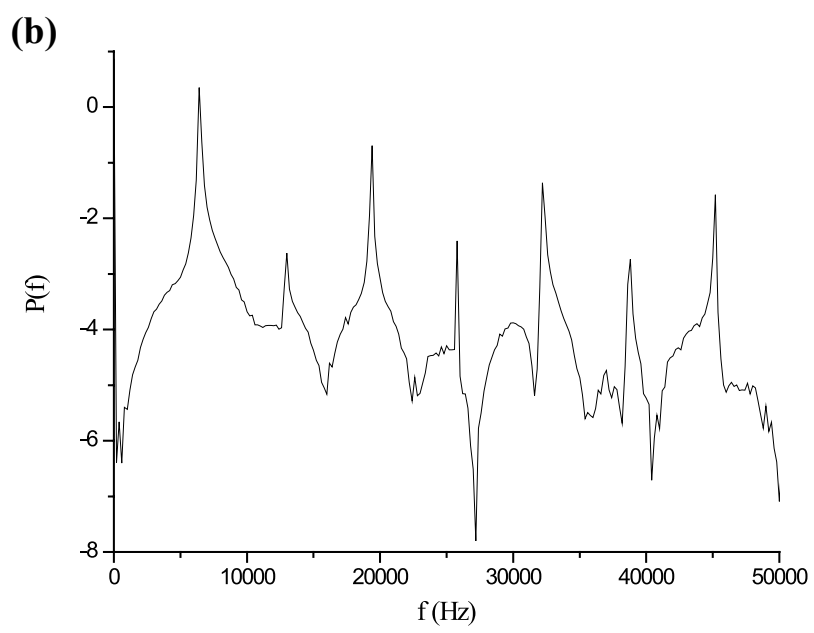

Figure 3. a) Digital input signal $M(t)$ and (b) Its power spectrum (FFT)
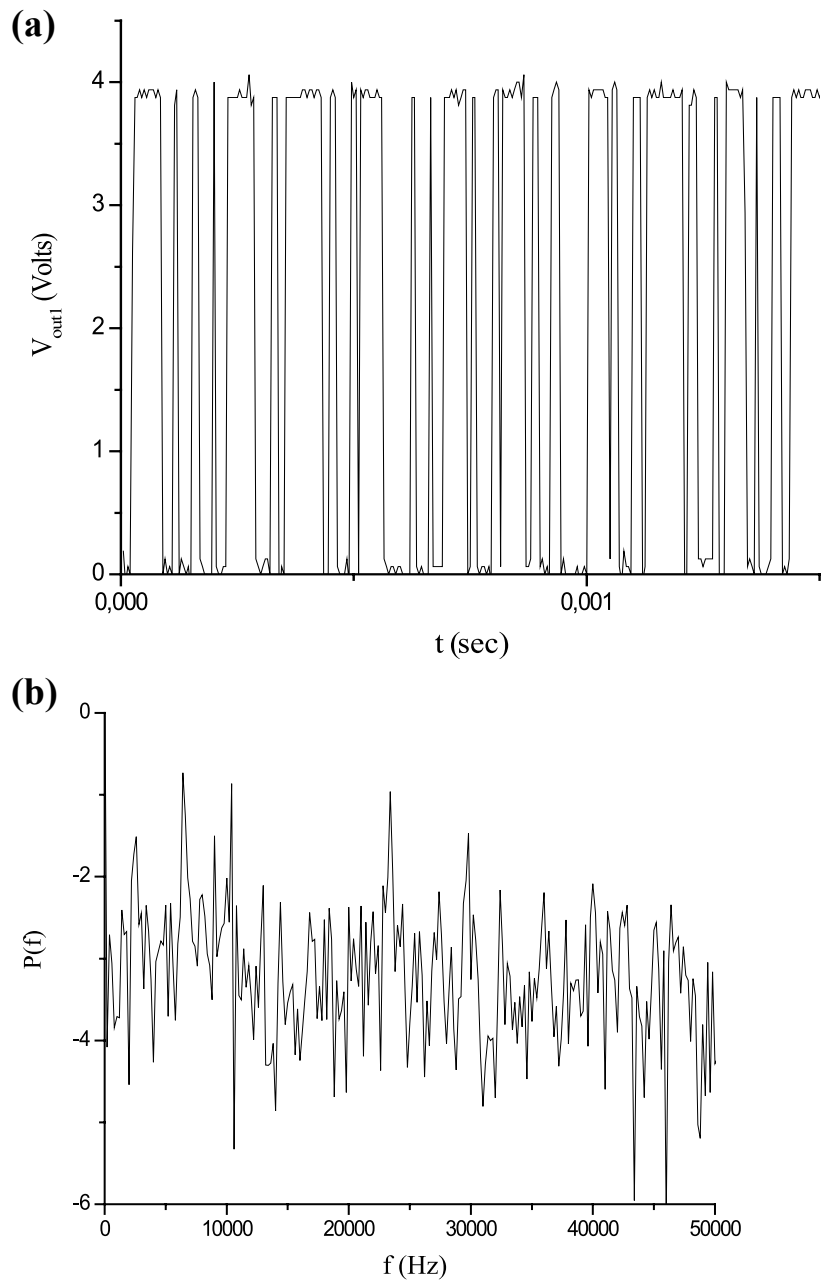

Figure 4. a) The transmitted chaotic signal $F(t)$ and (b) its power spectrum (FFT) 
(a)

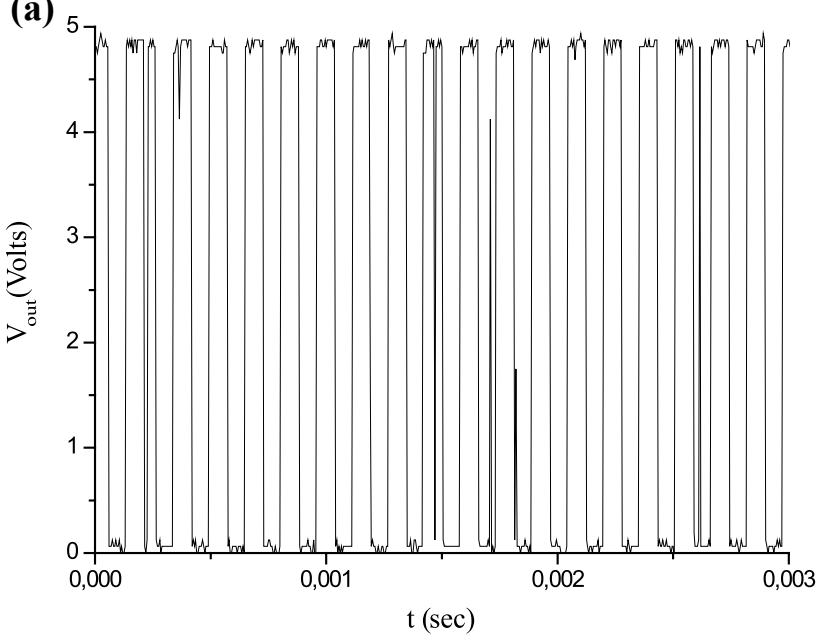

(b)

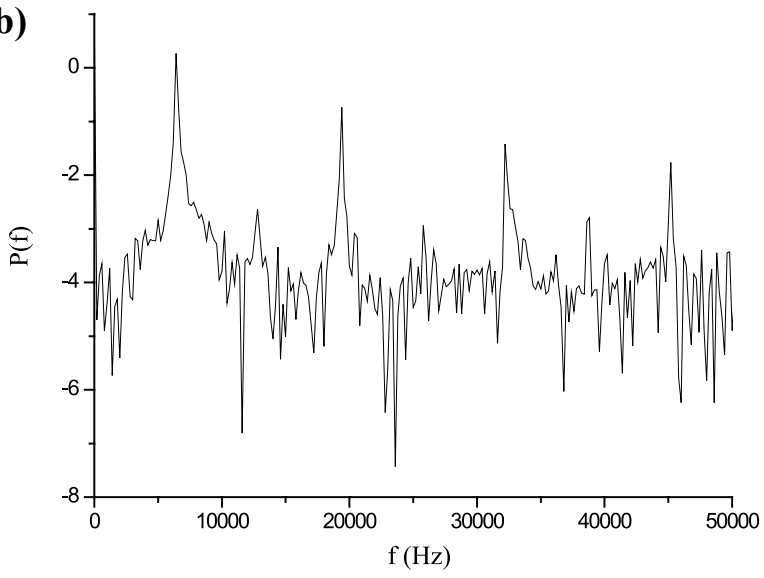

It should be mentioned, that the system security depends mainly on three major factors:

(a) The frequency of input signal $M(t)$, since it should be in the range that triggers the circuit to operate in a chaotic mode.

(b) The unfamiliarity of the topology used as a receiver circuit.

(c) The exact knowledge of all the parameters by any wouldbe intruder.

\section{Conclusions}

A scheme capable of secure chaotic digital communication is presented and experimentally studied. Both the information input signal and the transmitted chaotic signal, are discrete (digital) signals. The chaotic nature of the transmitted signal was verified by its power spectrum, while the transmitter's chaotic mode of operation was checked by its phase portrait. The recovered signal, as reconstructed after its synchronized demodulation in the receiver circuit, appears to be the same with the input pulse-series. This is confirmed by its power spectrum, which appears almost the same with the one of the input signal.

Acknowledgments: SGS, ANM, ANA and AV acknowledge support to NATO project ICS.EAP.CLG.983334, while SGS and LM acknowledge support to the Research and Fund Administration Committee of Kavala TEI (Act 7/5-12-2006 Subject no.1).

Figure 5. a) The recovered signal at the receiver's output and (b) its power spectrum (FFT)

\section{References}

1. M.P. Hanias, L. Magafas, "Time series cross prediction in a single transistor chaotic circuit using neural networks", J. Engin. Science Tech. Review, vol. 2, no. 1, pp. 8-11, 2009.

2. H.G. Shuster and W. Just, Deterministic Chaos: An Introduction, Weinhaim: WILEY-VCH, 2005.

3. T. Yang and L. Chua, "Impulsive stabilazation for control and synchronization of chaotic systems: Theory and application to secure communication”, IEEE Trans. Circuits Syst. I, vol.44, no.10, pp.976-988, Oct. 1997

4. See for instance: Proc. 12th Int. Workshop on Nonlinear Dynamics of Electronic Systems NDES'2004, Évora, Portugal, May 2004.

5. See for instance: Proc. 2005 IEEE International Symposium on Circuits and Systems ISCAS'2005, Kobe, Japan, May 2005.

6. T. Yang, "A survey of chaotic secure communication systems", Int. J. Comp. Cognition, vol. 2, pp. 81-130, June 2004.

7. J.C. Pizolato Jr, M.A. Romero and L.G. Neto, "Chaotic Comunication Based on the Particle-in-a-Box Electronic Circuit", IEEE Trans. CAS-I, vol. 55(4), pp. 11081115, May 2008.

8. G. Chen and T. Ueta, Chaos in Circuits and Systems, Singapore: World Scientific, 2002.

9. E. Ott, C. Grebogi and J.A. Yorke, "Controlling chaos", Phys. Rev. Lett., vol. 64, pp. 1196-1199, Mar. 1990.

10. E. Ott, Chaos in Dynamical Systems, Cambridge: Cambridge Univ. Press, 2002.
11. M.J. Ogorzalek, Chaos and Complexity in Nonlinear Electronic Circuits, Singapore, World Scientific, 1997.

12. L.M. Pecora, T.L. Carroll, "Synchronization in chaotic systems", Phys. Rev. Lett., vol. 64, pp. 821-824, Feb. 1990.

13. A. Pikovsky, M. Rosenblum and J. Kurths, Synchronization. A universal concept in nonlinear sciences, UK, Cambridge University Press, 2003.

14. C.W. Wu, Synchronization in Coupled Chaotic Circuits and Systems, World Scientific, 2002.

15. K.M. Cuomo and A.V. Oppenheim, Circuit implementation of synchronized chaos with applications to communications, Phys. Rev. Lett., 1993; 71: 65-68.

16. G. Kolumban, M.P. Kennedy and L.O. Chua, The role of synchronization in digital communications using chaos - part I: fundamentals of digital communications", IEEE Trans. Circuits Syst.-I, 1997; 44: 927-936.

17. A.N Miliou, A.P. Valaristos, S.G. Stavrinides, K.G. Kyritsi and A.N. Anagnostopoulos, Characterization of a non-autonomous second order nonlinear circuit for secure data transmission, Chaos Solitons \& Fractals, 2007; 33(4): 1248-1255.

18. A.N Miliou, I.P. Antoniades, S.G. Stavrinides and A.N. Anagnostopoulos, Secure communication by chaotic synchronization: Robustness under noisy conditions, Nonlinear Analysis: Real World Applications, 2007; 8: 1003-1012.

19. G. Mykolaitis, A. Tamasevicious, A. Cenys, A. Namajunas, K. Navionis 
and A. Anagnostopoulos, "Globally synchronizable non-autonomous chaotic oscillator", In Proc.7th Int. Workshop on Nonlinear Dynamics of Electronic Systems NDES'99, Rønne, Denmark, pp. 277-280, 1999.

20. S.G. Stavrinides, Characterization of the behavior of a nonlinear electronic oscillator producing chaotic signals, Phd Thesis, Aristotle University of Thessaloniki, 2007.

21. S.G. Stavrinides, K.G. Kyritsi, N.C. Deliolanis, A.N. Anagnostopoulos A. Tamaševičious, and A. Čenys, "The period doubling route to chaos of a second order non-linear non-autonomous chaotic oscillator - Part I", Chaos Solitons Fractals, vol. 20, pp. 849-854, May 2004.

22. S.G. Stavrinides, K.G. Kyritsi, N.C. Deliolanis, A.N. Anagnostopoulos, A. Tamaševičious, and A. Čenys, "The period doubling route to chaos of a second order non-linear non-autonomous chaotic oscillator - Part II", Chaos Solitons Fractals, vol. 20, pp. 843-847, May 2004.

23. S.G. Stavrinides, A.N Miliou, Th. Laopoulos, A.N. Anagnostopoulos, "The Intermittency route to chaos of a second order non-Linear non-autonomous oscillator", Int. J. on Bifurcation \& Chaos, vol. 18(5), pp. 15611566, 2008.
24. S.G. Stavrinides, N.C. Deliolanis, Th. Laopoulos, I.M. Kyprianidis, A. N. Miliou and A. N. Anagnostopoulos, "The intermittent behavior of a second order non-linear non-autonomous oscillator", Chaos Solitons and Fractals, 36(5), pp. 1191-1199, 2008.

25. S.G. Stavrinides, N.C. Deliolanis, A.N Miliou, Th. Laopoulos, and A.N. Anagnostopoulos, "Internal crisis in a second order non-linear non-autonomous electronic oscillator", Chaos Solitons \& Fractals, 36(4), pp. 1055 1061, 2008.

26. S.G. Stavrinides, Th. Laopoulos, A.N. Anagnostopoulos, "An Automated Acquisition Setup for the analysis of chaotic systems", In Proc. IEEE IDAACS, pp. 628-632, Sofia, Bulgaria, 2005.

27. G. Perez and H.A. Cerdeira, "Extracting messages masked by chaos", Phys. Rev. Lett., vol. 74, pp. 1970-1973, Mar. 1995.

28. C. Tao, G. Du and Y. Zhang, "Decoding digital information from the cascaded heterogeneous chaotic systems", Int. J. Bifurc. Chaos, vol. 13(6), pp. 1599-1608, 2003. 